\title{
Assessment of Groundnut (Arachis hypogaea L.) Mini Core for Resistance to Multiple Biotic Stresses under Hot Spot Location
}

\author{
A. Mohammad Saleem, K. Gopalakrishna Naidu* , H.L. Nadaf and P.S. Tippannavar \\ AICRP on Groundnut, Main Agriculture Research Station, University of Agricultural \\ Sciences, Dharwad - 580 005, Karnataka, India \\ *Corresponding author
}

\section{A B S T R A C T}

Groundnut is one of the important oilseed crops of India. Several biotic stresses affect in achieving the real potential yields. Cultivation of biotic stress resistant cultivars is an

\section{Keywords}

Groundnut, Mini core, Spodoptera litura, Late leaf spot, Rust

\section{Article Info}

Accepted:

12 October 2018

Available Online:

10 November 2018 ecologically and economically sound approach. The occurrence of different biotic stresses varies with location and season thus limiting the wide adoption of varieties resistant to one or the other biotic stresses. This necessitates the importance of evolving multiple biotic stress resistant genotypes. In the present study, a set of 184 mini core genotypes along with 12 checks were assessed for major foliar diseases late leaf spot and rust and a major defoliator, Spodoptera litura in hot spot location. In mini core, hypogaea botanical group had higher frequency of resistant genotypes to Spodoptera litura (31\%), late leaf spot (33 $\%$ ) and rust (8\%) when compared to other botanical groups. Among the Spodoptera litura resistant genotypes, eight genotypes viz., ICG 862, ICG 928, ICG 76, ICG 2777, ICG 5016, ICG 12276, ICG 4412 and ICG 9905 had significantly higher pod yield per plant (> $23.2 \mathrm{~g})$ compared to check JL $24(18.1 \mathrm{~g})$. Among the 184 genotypes of mini core, five genotypes viz., ICG 2381, ICG 11426, ICG 12625, ICG 12370 and ICG 875 were resistant to late leaf spot (field disease score < 4). ICG 2381 had resistance to late leaf spot, rust and Spodoptera litura but had low pod yield per plant and hence can serve as potential multiple stress resistant donor genotype in breeding programmes.

\section{Introduction}

Groundnut, an important oil seed crop is cultivated in more than 100 countries in an area of 27.66 mha with an annual production of $43.98 \mathrm{mt}$ and productivity of $1590 \mathrm{~kg} \mathrm{ha}^{-1}$ (FAO, 2016). In India, groundnut is grown in an area of $5.80 \mathrm{~m}$ ha, with production of 6.85 $\mathrm{m} \mathrm{t}$ and productivity of $1182 \mathrm{~kg} \mathrm{ha}^{-1}$ (FAO, 2016). Gujarat and Andhra Pradesh rank first and second, respectively both in area and production followed by Karnataka in area and
Tamil Nadu in production. Though, India is a leading producer of groundnut, its productivity is low $\left(1182 \mathrm{~kg} \mathrm{ha}^{-1}\right)$ compared to USA (4118 $\left.\mathrm{kg} \mathrm{ha}^{-1}\right)$, China $\left(3674 \mathrm{~kg} \mathrm{ha}^{-1}\right)$ and Argentina $\left(2928 \mathrm{~kg} \mathrm{ha}^{-1}\right)(\mathrm{FAO}, 2016)$, the reasons being lack of improved high yielding cultivars, cultivation under shallow soils of low fertility, uneven rainfall distribution, continuous cropping without crop rotation, low plant population, incidence of biotic and abiotic stresses. Among the various biotic stresses affecting the groundnut crop, tobacco 
cutworm (Spodoptera litura), late leaf spot and rust have major role in reducing the yield levels to a considerable extent. Spodoptera litura (F.) is considered as a pest of national importance and yield losses are reported to be 13-71 per cent in the states of Karnataka and Andhra Pradesh (Amin, 1983). In India, transitional tract of Karnataka (Dharwad) has been identified as hot spot for Spodoptera litura during kharifseason, where yield loss to the extent of 66.6 per cent was reported in groundnut (Kulkarni, 1989).

Among the foliar diseases, late leaf spotand rust were more threatening than other diseases. The pod loss of 50-80 per cent due to rust was reported in an epidemic year (Sandhikar et al., 1989). Late leaf spot can cause a reduction of $10-50$ per cent yield (McDonald et al., 1985). In transitional tract of Karnataka, late leaf spot resulted in yield loss up to 50 per cent (Puranik et al., 1973; Astaputre and Kulkarni, 1996). Late leaf spot and rust occur together commonly whenever the groundnut is cultivated, but their incidence and damaging level is different with respect to location and season. Both the diseases have individual capacity to cause economic level of yield loss. In India, late leaf spot and rust occur together and cause yield loss up to 70 per cent (Subrahmanyam et al., 1990).

Though many effective chemicals are suggested to control Spodoptera litura, late leaf spot and rust, but are not eco-friendly and add to the cost of cultivation. In this regard, breeding for inbuilt resistance occupies importance and is an amenable approach which necessitates the identification of potential resistant sources. Earlier, mutant 45, NC Ac 343, mutant 28-2 (Prasad and Gowda, 2006), ICGV 91180, NC Ac 343, M 28-2 and M 45 (Naidu et al., 2016) were identified as resistant to Spodoptera litura with minimum leaf damage. Dwivedi et al., (2002) reported ICGV 99005, 99003, 99012 and 99015 as rust resistant while, ICGV 99006, 99013, 99004, 99003 and 99001 as resistant to late leaf spot under artificial condition. GPBD 4, B 37c and ICGV 87165 were resistant to both late leaf spot and rust with significantly lower field disease score and AUDPC (Motagi et al., 2014). Sudini et al., (2015) reported that ICGs 11426, 11088, 4389, 6022, 6993, 2857, 4746, $15419,6402,6766$ were resistant to rust and ICG's 14127, 5051, 6646, 7153, 12276, 12625, 2925, 9961, 10036 were moderately resistant to late leaf spot and also reported that none were found resistant to both late leaf spot and rust.

Groundnut mini core was evaluated for late leaf spot and rust resistance (Yugandhar, 2005; Madhura, 2006, Shivaleela, 2012; Rajashekar, 2013; Upadhyaya et al., 2014, Sudhini et al., 2015). But, no study is reported on evaluation for Spodoptera litura resistance. Present study is aimed at assessing of resistance to Spodoptera litura in addition to major foliar diseases late leaf spot and rust under hot spot location and identification of multiple resistant genotypes in groundnut mini core.

\section{Materials and Methods}

The study comprised groundnut mini core having 184 genotypes, a subset of core germplasm and was collected from ICRISAT, Hyderabad. In addition to this, four control genotypes (ICG 11457, ICG 12370, ICG 13099 and ICG 13723), three susceptible (JL 24, TMV 2, TAG 24) and five resistant genotypes (ICGV 86031, ICGV 87157, ICGV 87160, ICG 2271 and ICG 1657) as checks to different biotic stresses were included in the study. The study was conducted at Main Agriculture Research Station (MARS), University of Agriculture Sciences, Dharwad, India $\left(15^{\circ} 13^{\prime} \mathrm{N}, 75^{\circ} 07^{\prime} \mathrm{E}, 678 \mathrm{~m}\right.$ above mean sea level, and $800 \mathrm{~mm}$ average annual rainfall) during rainy season 2017 . 
Each genotype was sown in a row of 2-meter length with 2 replications in 4 blocks by following randomized incomplete block design. Spanish and Virginia bunch genotypes were sown in $30 \times 10 \mathrm{~cm}$ spacing while, Virginia runner genotypes in $60 \times 10 \mathrm{~cm}$ spacing. The susceptible check JL 24 for all the three biotic stresses was planted after every 10 test entries to assure maximum incidence. Normal package of practices were followed to raise the healthy crop avoiding the plant protection measures. These genotypes were evaluated for their reaction to foliar diseases viz., late leaf spot and rust and defoliator pest Spodoptera litura under hot spot conditions for all the three biotic stresses at Dharwad (Karnataka). In addition, they were assessed for various morphological and productivity traits.

\section{Evaluation of mini core genotypes for various biotic stresses}

\section{Spodoptera litura}

Visual observations on per cent leaf damage due to $S$. litura (0-100\%) were recorded at 70 days after sowing (coinciding with peak incidence of the insect pest) by following the standard scale (0-9) as shown in plate 1 (Anon., 2015). The observation on per cent leaf damage was assessed by leaf damage at top, middle and bottom leaves from 5 plants showing maximum incidence of insect in each genotype and expressed as per cent leaf damage.

\section{Late Leaf Spot (LLS) and rust}

The evaluation of genotypes against late leaf spot (LLS) and rust was taken at 80 days after sowing (DAS) which was coinciding with highest incidence as evident by highest field disease score in susceptible genotypes for both LLS and rust. The five plants in each genotype based on highest disease incidence were assessed for damage due to late leaf spot and rust. The observation on per cent leaf area damage by pathogen of rust and late leaf spot was assessed by following the modified 9point scale (Subrahamanyam et al., 1995).

\section{Statistical analysis}

Analysis of variance and correlation was carried out using GenStat regression method, as the experiment contained both spreading and bunch type genotypes which led to unequal number of genotypes in each block.

\section{Results and Discussion}

\section{Variability for different biotic stresses}

Analysis of variance for reaction to biotic stresses (Spodoptera litura, late leaf spot and rust) and productivity parameters (days to initiation of flowering, days to 50 per cent flowering, plant height, number of primary branches per plant, number of pods per plant, shelling per cent, hundred seed weight and pod yield per plant) in the mini core indicated highly significant genotypic differences for these traits (Table 1) which is pre-requisite for genetic improvement through plant breeding.

The difference between the phenotypic and genotypic coefficient of variation was very low for reaction to different biotic stresses and productivity parameters (Table 2) indicating the predominance of genetic component in governing these traits. The extent of genotypic variability was high for response to Spodoptera litura and pod yield per plant in mini core genotypes indicating scope for selection of genotypes with resistance to Spodoptera litura and also for pod yield per plant in this material.

The extent of genotypic variability was also high for reaction to late leaf spot and rust in case of mini core genotypes (Table 2). The 
results of Khedikar (2008), Khote et al., (2009) and Savaliya et al., (2009) also indicated higher variability for late leaf spot and rust. On the contrary, there was less genotypic variability for days to initiation of flowering, days to fifty per cent flowering and shelling per cent in mini core genotypes revealing very less scope for identification of superior genotypes for these traits. Such low genetic variation was reported earlier by Venkataramana et al., (2001), Nadaf and Habib (1987), Sharma and Gupta (2011) for shelling percentage in groundnut.

High heritability coupled with high genetic advance for response to Spodoptera litura, late leaf spot and rust and for number of pods per plant, hundred seed weight and yield per plant among the productivity parameters in the mini core genotypes (Table 2) revealed relatively higher additive component of genetic variance and hence genetic improvement for these traits would be possible through simple selection based on phenotype (Painwadee et al., 2009).

Earlier, Nath and Alam (2002), Venkataraman (2001), Apte et al., (2008), Raut et al., (2010) and Rao et al., (2012) also reported higher additive components of genetic variation for late leaf spot, rust, number of pods per plant and yield per plant.

The indirect selection for yield through yield components like number of pods per plant which has high heritability seems to be much more rewarding than direct selection for yield alone. Shelling per cent on the other hand, had low genetic advance over mean in mini core and elite cultivars because of its low level of heritability and variability. Even though days to initiation of flowering and days to fifty per cent flowering exhibited high level of heritability, genetic advance over mean was low because of very low magnitude of genetic variation indicating very less scope of selection for these traits.

\section{Correlation between different biotic stresses and productivity parameters}

There was a significant positive association between Spodoptera litura damage and late leaf spot incidence in groundnut mini core (Table 3) indicating that the genotypes that were susceptible to Spodoptera litura were also susceptible to late leaf spot. Prasad (1997) also reported positive association between Spodoptera litura damage and late leaf spot incidence in the groundnut mutants. Spodoptera litura damage had significant negative correlation with days to initiation of flowering and days to fifty per cent flowering in mini core genotypes revealing late flowering and maturing nature of biotic stress resistant genotypes. Naidu (2002) reported that majority of interspecific derivatives matured late which were showing resistance to biotic stresses.

Spodoptera litura damage had significant negative correlation with hundred seed weight in mini core indicating that there would be reduction in seed size in the Spodoptera litura susceptible genotypes. Spodoptera litura damage had non-significant correlation with yield per plant in the mini core germplasm suggesting that Spodoptera litura damage does not have major impact on yield which could be occurrence of Spodoptera (70 days after sowing) and hence not having too much cost on yield.

Significant negative correlation was observed between late leaf spot and pod yield per plant in mini core germplasm indicating susceptibility to late leaf spot has more cost on yield in groundnut. The results were in conformity with those of Motagi (2001) who has reported negative correlation between late leaf spot and rust resistance with pod yield while studying mutants. Motagi et al., (1997) reported negative association of late leaf spot with yield in Spanish bunch mutants. 
Plate.1 Leaf damage for visual scoring of Spodoptera litura damage in groundnut

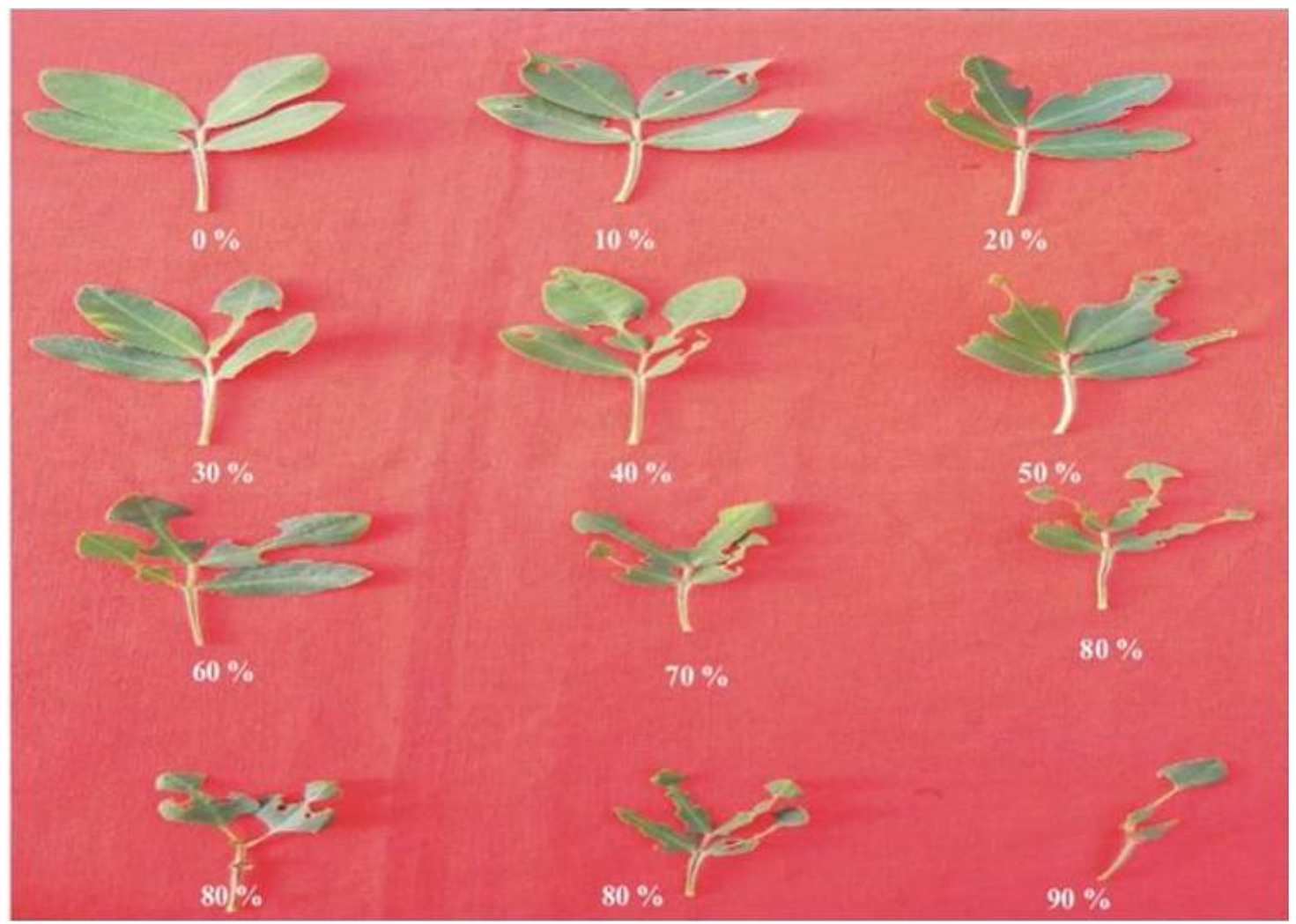

Table.1 Mean sum of squares for various biotic stresses and productivity parameters in mini core germplasm during kharif 2017

\begin{tabular}{l} 
Phenotypic traits \\
\hline \multicolumn{1}{c|}{ df } \\
\hline Leaf damage by Spodoptera litura (\%) at \\
70 DAS \\
\hline Late leaf spot at 80 DAS \\
\hline Rust at 80 DAS \\
\hline Days to initiation of flowering \\
\hline Days to $50 \%$ flowering \\
\hline Plant height (cm) \\
\hline Number of primary branches per plant \\
\hline Number of pods per plant \\
\hline Shelling per cent \\
\hline Hundred seed weight (g) \\
\hline Yield per plant (g) \\
\hline
\end{tabular}

\begin{tabular}{|c|c|c|c|}
\hline Replication & $\begin{array}{c}|c| \\
\text { Blocks within } \\
\text { replication }\end{array}$ & Genotype & Error \\
\hline 1 & 6 & 193 & 191 \\
\hline 16.26 & $342.98 * *$ & $126.40 * *$ & 5.76 \\
\hline 0.09 & $31.33 * *$ & $2.31 * *$ & 0.33 \\
\hline 0.82 & $1.94 * *$ & $1.25 * *$ & 0.34 \\
\hline 0.04 & $96.16 * *$ & $4.09 * *$ & 0.35 \\
\hline 0.25 & $91.00 * *$ & $3.95 * *$ & 0.52 \\
\hline 6.35 & $801.37 * *$ & $64.75 * *$ & 4.27 \\
\hline 0.16 & $92.47 * *$ & $8.16 * *$ & 0.09 \\
\hline 2.83 & $249.46 * *$ & $35.04 * *$ & 0.74 \\
\hline 2.64 & $125.25 * *$ & $31.48 * *$ & 0.52 \\
\hline 3.90 & $180.59 * *$ & $62.82 * *$ & 3.00 \\
\hline 4.75 & $455.59 * *$ & $47.90 * *$ & 2.93 \\
\hline
\end{tabular}

$* \& * *$ - Significant at 5 and 1 per cent level of probability, respectively 


\section{Int.J.Curr.Microbiol.App.Sci (2018) 7(11): 1599-1614}

Table.2 Genetic components of variation for various biotic stresses and productivity parameters in mini core germplasm

\begin{tabular}{|c|c|c|c|c|c|c|c|c|}
\hline Phenotypic traits / Components & Minimum & Maximum & Mean & PCV $(\%)$ & GCV $(\%)$ & $\mathbf{h}^{2}$ bs & GA & GAM \\
\hline $\begin{array}{l}\text { Leaf damage by Spodoptera litura (\%) at } \\
70 \text { DAS }\end{array}$ & 4.5 & 45.0 & 19.1 & 44.0 & 42.0 & 91.1 & 15.7 & 82.6 \\
\hline Late leaf spot at 80 DAS & 2.5 & 8.0 & 6.5 & 20.5 & 18.2 & 79.3 & 2.1 & 33.5 \\
\hline Rust at 80 DAS & 2.0 & 7.0 & 6.23 & 15.8 & 14.2 & 80.2 & 1.6 & 26.3 \\
\hline Days to initiation of flowering & 26 & 34.5 & 29.8 & 6.4 & 6.0 & 89.9 & 3.5 & 11.9 \\
\hline Days to fifty per cent flowering & 27.5 & 36.0 & 31.6 & 6.0 & 5.5 & 85.2 & 3.3 & 10.5 \\
\hline Plant height (cm) & 14.3 & 55.5 & 30.9 & 22.0 & 21.0 & 90.6 & 12.7 & 41.2 \\
\hline Number of primary branches per plant & 4.1 & 14 & 6.7 & 35.5 & 34.8 & 95.9 & 4.7 & 70.2 \\
\hline Number of pods per plant & 8.3 & 33.0 & 16.6 & 28.4 & 26.5 & 87.2 & 8.5 & 51.2 \\
\hline Shelling per cent & 54.4 & 76.7 & 69.2 & 6.0 & 5.0 & 70.4 & 6.0 & 8.7 \\
\hline Hundred seed weight (g) & 24.6 & 55.2 & 38.6 & 15.3 & 14.6 & 90.1 & 11.0 & 28.5 \\
\hline Yield per plant (g) & 4.8 & 33.4 & 14.7 & 39.3 & 35.7 & 80.8 & 9.6 & 65.5 \\
\hline
\end{tabular}

PCV-Phenotypic co-efficient of variation $(\%)$

GA- Genetic advance

GCV- Genotypic co-efficient of variation (\%)

$\mathrm{h}_{\mathrm{bs}}^{2}$ - Heritability (Broad sense)
GAM- Genetic advance as per cent of mean 
Table.3 Phenotypic and genotypic correlation among various biotic stresses and productivity parameters in mini core germplasm

\begin{tabular}{|c|c|c|c|c|c|c|c|c|c|c|c|}
\hline Traits & $\begin{array}{c}\text { Spodoptera } \\
\text { litura } \\
\text { damage }\end{array}$ & $\begin{array}{l}\text { Late } \\
\text { leaf } \\
\text { spot }\end{array}$ & Rust & $\begin{array}{c}\text { Days to } \\
\text { initiation } \\
\text { of } \\
\text { flowering }\end{array}$ & $\begin{array}{l}\text { Days to } \\
\text { fifty per } \\
\text { cent } \\
\text { flowering }\end{array}$ & $\begin{array}{l}\text { Plant } \\
\text { height }\end{array}$ & $\begin{array}{l}\text { Number of } \\
\text { primary } \\
\text { branches } \\
\text { per plant }\end{array}$ & $\begin{array}{l}\text { Number } \\
\text { of pods } \\
\text { per plant }\end{array}$ & $\begin{array}{l}\text { Shelling } \\
\text { per } \\
\text { cent }\end{array}$ & $\begin{array}{l}\text { Hundred } \\
\text { seed } \\
\text { weight }\end{array}$ & $\begin{array}{l}\text { Yield } \\
\text { per } \\
\text { plant }\end{array}$ \\
\hline $\begin{array}{l}\text { Spodoptera litura } \\
\text { damage }\end{array}$ & 1 & $0.462 * *$ & $0.162 * *$ & $-0.434 * *$ & $-0.439 * *$ & $0.179 * *$ & $-0.352 * *$ & -0.028 & -0.010 & $-0.198 * *$ & -0.067 \\
\hline Late leaf spot & $0.543 * *$ & 1 & $0.201 * *$ & $-0.636 * *$ & $-0.595 * *$ & $0.306^{* *}$ & $-0.476^{* *}$ & $-0.185 * *$ & -0.025 & -0.050 & $-0.223 * *$ \\
\hline Rust & $0.207 * *$ & $0.280 * *$ & 1 & -0.067 & 0.055 & -0.055 & -0.044 & -0.020 & 0.095 & -0.050 & 0.071 \\
\hline $\begin{array}{l}\text { Days to initiation } \\
\text { of flowering }\end{array}$ & $-0.476 * *$ & $-0.727 * *$ & -0.087 & 1 & $0.960 * *$ & $-0.377 * *$ & $0.555^{* *}$ & $0.220 * *$ & -0.067 & $0.231 * *$ & $0.220 * *$ \\
\hline $\begin{array}{l}\text { Days to fifty per } \\
\text { cent flowering }\end{array}$ & $-0.485^{* *}$ & $-0.703 * *$ & -0.081 & $0.991 * *$ & 1 & $-0.370 * *$ & $0.554 * *$ & $0.227 * *$ & -0.063 & $0.232 * *$ & $0.215^{* *}$ \\
\hline Plant height & $0.192 * *$ & $0.348 * *$ & -0.083 & $-0.418 * *$ & $-0.425 * *$ & 1 & $-0.323 * *$ & $-0.293 * *$ & 0.032 & 0.098 & $-0.205^{* *}$ \\
\hline $\begin{array}{l}\text { Number of } \\
\text { primary } \\
\text { branches per } \\
\text { plant }\end{array}$ & $0.457 * *$ & $-0.544 * *$ & -0.061 & $0.593 * *$ & $0.614 * *$ & $-0.336 * *$ & 1 & $0.421 * *$ & -0.089 & $0.198 * *$ & $0.195 * *$ \\
\hline $\begin{array}{l}\text { Number of pods } \\
\text { per plant }\end{array}$ & -0.032 & $-0.241 * *$ & -0.065 & $0.264 * *$ & $0.273 * *_{-}$ & $0.329 * *$ & $0.457 * *$ & 1 & 0.053 & 0.041 & $0.219 * *$ \\
\hline Shelling per cent & -0.036 & -0.024 & 0.093 & -0.099 & -0.097 & 0.056 & -0.056 & 0.086 & 1 & $0.106^{*}$ & 0.051 \\
\hline $\begin{array}{l}\text { Hundred seed } \\
\text { weight }\end{array}$ & $-0.224 * *$ & $-0.316^{* *}$ & $-0.134 * *$ & $0.260 * *$ & $0.278 * *$ & $0.115^{*}$ & $0.215^{* *}$ & 0.042 & $0.148 * *$ & 1 & $0.152 * *$ \\
\hline Yield per plant & -0.063 & $-0.258 * *$ & 0.094 & $0.263^{* *}$ & $0.274 * *$ & $-0.219 * *$ & $0.199 * *$ & $0.246 * *$ & 0.063 & $0.194 * *$ & 1 \\
\hline
\end{tabular}

*\&**- Significant at 5 and 1 per cent level of probability, respectively

Values above the diagonal represents phenotypic correlation while values below the diagonal represents the genotypic correlation. 
Table.4 Mean performance of mini core genotypes showing $\leq 10$ per cent Spodoptera litura damage (at 70 DAS)

\begin{tabular}{|c|c|c|c|c|c|c|c|c|c|c|c|c|c|}
\hline $\begin{array}{l}\text { Sl. } \\
\text { No. }\end{array}$ & Genotypes & $\begin{array}{l}\text { Botanical } \\
\text { variety }\end{array}$ & $\begin{array}{c}\text { Spodoptera } \\
\text { damage }(\%)\end{array}$ & $\begin{array}{l}\text { Late } \\
\text { leaf } \\
\text { spot }\end{array}$ & Rust & $\begin{array}{l}\text { Days to } \\
\text { initiation } \\
\text { of } \\
\text { flowering }\end{array}$ & $\begin{array}{c}\text { Days to } \\
50 \% \\
\text { flowering }\end{array}$ & $\begin{array}{l}\text { Plant } \\
\text { height } \\
(\mathrm{cm})\end{array}$ & $\begin{array}{c}\text { No. of } \\
\text { primary } \\
\text { branches } \\
\text { per } \\
\text { plant }\end{array}$ & $\begin{array}{l}\text { No. of } \\
\text { pods } \\
\text { per } \\
\text { plant }\end{array}$ & $\begin{array}{c}\text { Shelling } \\
\text { per } \\
\text { cent }\end{array}$ & $\begin{array}{l}\text { Hundred } \\
\text { seed } \\
\text { weight } \\
\text { (g) }\end{array}$ & $\begin{array}{l}\text { Yield } \\
\text { per } \\
\text { plant } \\
(\mathrm{g})\end{array}$ \\
\hline 1 & ICG 862 & hypogaea & 4.5 & 4.5 & 6.5 & 32.5 & 33.5 & 20.8 & $8.4 * *$ & $13.1^{*}$ & $72.6 * *$ & 33.9 & $23.2 *$ \\
\hline 2 & ICG 928 & hypogaea & 5.0 & 4.5 & 7.0 & 32.5 & 34.5 & 26.7 & $8.5 * *$ & $18.6 * *$ & $71.9 *$ & 42.7 & $28.2 * *$ \\
\hline 3 & ICG 5051 & hypogaea & 5.4 & 7.0 & 7.0 & $28.0 *$ & $30.0^{*}$ & 20.0 & 4.8 & 10.2 & $73.4 * *$ & 36.7 & 14.4 \\
\hline 4 & ICG 2925 & hypogaea & 6.7 & 6.5 & 6.5 & 32.0 & 34.0 & 18.0 & $9.0 * *$ & $20.9 * *$ & 67.5 & 32.6 & 11.4 \\
\hline 5 & ICG 14523 & hypogaea & 6.8 & 5.5 & 5.5 & 32.0 & 33.5 & 29.7 & $9.4 * *$ & $15.4 * *$ & $70.9 *$ & 40.7 & 15.3 \\
\hline 6 & ICG 76 & hypogaea & 6.8 & 5.0 & 6.5 & 33.5 & 34.5 & 29.3 & $7.7 * *$ & $13.5 * *$ & $72.9 * *$ & $51.5^{* *}$ & $25.2 * *$ \\
\hline 7 & ICG 10890 & fastigiata & 7.5 & 5.5 & 6.5 & 28.5 & $30.0 *$ & 30.1 & 5.0 & $12.3 *$ & 62.1 & 25.1 & 14.8 \\
\hline 8 & ICG 14705 & hypogaea & 7.5 & 6.5 & 6.5 & 28.5 & 31.5 & 27.6 & 6.2 & $13.7 * *$ & 72.6 & 35.0 & 9.3 \\
\hline 9 & ICG 2777 & hypogaea & 7.7 & 5.0 & 6.5 & 33.5 & 35.5 & 21.4 & $8.6 * *$ & $29.3 * *$ & 70.1 & 33.4 & $35.2 * *$ \\
\hline 10 & ICG 14466 & hypogaea & 7.8 & 6.0 & 5.5 & 30.0 & 32.0 & 28.1 & $8.1 * *$ & $13.6 * *$ & 69.6 & 37.3 & 9.6 \\
\hline 11 & ICG 13787 & hypogaea & 7.8 & 6.0 & 7.0 & 32.0 & 33.5 & 27.0 & $9.3^{* *}$ & $17.2 * *$ & 67.9 & $45.3 * *$ & 14.3 \\
\hline 12 & ICG 9777 & hypogaea & 8.1 & 7.0 & 6.5 & 32.5 & 34.5 & 31.8 & 5.8 & 11.7 & $70.9 *$ & 43.0 & 12.7 \\
\hline 13 & ICG 5016 & hypogaea & 8.2 & 4.5 & 5.5 & 32.0 & 34.0 & 25.7 & $10.6 * *$ & $16.8 * *$ & $71.8 *$ & 42.0 & $25.2 * *$ \\
\hline 14 & ICG 1668 & hypogaea & 8.3 & 5.5 & 5.5 & 31.5 & 33.5 & 32.0 & $11.4 * *$ & $13.9 * *$ & 54.5 & $46.9 * *$ & 12.5 \\
\hline 15 & ICG 5662 & hypogaea & 8.3 & 6.5 & 5.5 & 31.5 & 33.5 & 28.0 & $7.8 * *$ & $17.6 * *$ & 67.1 & 40.9 & 12.0 \\
\hline 16 & ICG 14482 & hypogaea & 8.3 & 5.5 & 6.5 & 31.5 & 33.5 & 38.1 & $8.2 * *$ & $13.3^{*}$ & 70.0 & $47.6 * *$ & 12.6 \\
\hline 17 & ICG 12276 & hypogaea & 8.4 & 5.0 & 5.5 & 29.0 & 31.5 & 32.9 & $8.2 * *$ & $17.0 * *$ & 73.4 & 43.4 & $26.3 * *$ \\
\hline 18 & ICG 2381 & hypogaea & 8.8 & 2.5 & 2.5 & 31.5 & 33.0 & 29.6 & $9.4 * *$ & $23.9 * *$ & 69.0 & $47.6 * *$ & 9.3 \\
\hline
\end{tabular}


Contd......

\begin{tabular}{|c|c|c|c|c|c|c|c|c|c|c|c|c|c|}
\hline $\begin{array}{l}\text { SI. } \\
\text { No. }\end{array}$ & Genotypes & $\begin{array}{c}\text { Botanical } \\
\text { variety }\end{array}$ & $\begin{array}{c}\text { Spodoptera } \\
\text { damage } \\
(\%)\end{array}$ & $\begin{array}{l}\text { Late } \\
\text { leaf } \\
\text { spot }\end{array}$ & Rust & $\begin{array}{c}\text { Days to } \\
\text { initiation } \\
\text { of } \\
\text { flowering }\end{array}$ & $\begin{array}{c}\text { Days to } \\
50 \% \\
\text { flowering }\end{array}$ & $\begin{array}{c}\text { Plant } \\
\text { height } \\
(\mathrm{cm})\end{array}$ & $\begin{array}{c}\text { No. of } \\
\text { primary } \\
\text { branches } \\
\text { per } \\
\text { plant }\end{array}$ & $\begin{array}{c}\text { No. of } \\
\text { pods } \\
\text { per } \\
\text { plant }\end{array}$ & $\begin{array}{c}\text { Shelling } \\
\text { per } \\
\text { cent }\end{array}$ & $\begin{array}{l}\text { Hundred } \\
\text { seed } \\
\text { weight } \\
\text { (g) }\end{array}$ & $\begin{array}{l}\text { Yield } \\
\text { per } \\
\text { plant } \\
\text { (g) }\end{array}$ \\
\hline 19 & ICG 4412 & hypogaea & 9.0 & 4.5 & 5.5 & 32.5 & 34.5 & 23.5 & $13.1 * *$ & $33.1 * *$ & 69.5 & $48.1 * *$ & $26.2 * *$ \\
\hline 20 & ICG 4538 & hypogaea & 9.3 & 6.5 & 6.0 & 31.5 & 32.5 & 31.3 & $14.9 * *$ & $28.6^{* *}$ & 68.1 & $44.3 * *$ & 13.3 \\
\hline 21 & ICG 9905 & hypogaea & 9.3 & 4.5 & 6.5 & 32.5 & 34.0 & 31.0 & $9.5 * *$ & $14.9 * *$ & 67.4 & 39.8 & $27.0 * *$ \\
\hline 22 & ICG 11855 & hypogaea & 9.5 & 6.5 & 6.5 & 30.5 & 32.0 & 31.6 & $13.9 * *$ & $23.5 * *$ & 65.6 & $51.7 * *$ & 20.9 \\
\hline 23 & ICG 2511 & hypogaea & 9.5 & 6.5 & 6.5 & 32.5 & 34.5 & 23.3 & $8.8 * *$ & $21.6 * *$ & 67.7 & 32.6 & 21.6 \\
\hline 24 & ICG 513 & hypogaea & 9.6 & 6.0 & 5.5 & $28.5^{*}$ & $30.0^{*}$ & 36.1 & 4.7 & 11.8 & 63.0 & $54.4 * *$ & 7.3 \\
\hline 25 & ICG 6913 & hypogaea & 9.7 & 5.5 & 6.5 & 31.5 & 33.5 & 26.9 & $8.7 * *$ & $15.9 * *$ & 69.9 & $46.6^{* *}$ & 9.2 \\
\hline 26 & ICG 15419 & hirsuta & 9.7 & 4.5 & 5.5 & 32.0 & 33.5 & 26.7 & $8.1 * *$ & $12.6^{*}$ & $73.6 * *$ & $43.1 *$ & 16.8 \\
\hline 27 & ICG 4746 & hypogaea & 9.8 & 7.0 & 6.0 & 30.5 & 32.0 & 25.1 & $7.7 * *$ & $17.1 * *$ & 64.0 & 33.6 & 9.7 \\
\hline 28 & ICG 7000 & hypogaea & 9.8 & 6.5 & 6.5 & 30.5 & 32.0 & 39.1 & 4.9 & 12.3 & 65.1 & $45.1 * *$ & 11.9 \\
\hline \multirow[t]{2}{*}{29} & ICG 721 & hyроваеа & 9.9 & 5.5 & 6.0 & 31.5 & 32.5 & 44.2 & $8.9 * *$ & $14.8 * *$ & 66.3 & 39.2 & 6.0 \\
\hline & Checks & & & & & & & & & & & & \\
\hline 30 & JL 24 & fastigiata & 43.4 & 7.5 & 6.5 & 29.0 & $30.5^{*}$ & 34.5 & 5.4 & 9.0 & 66.5 & 40.0 & 18.1 \\
\hline 31 & ICG 2271 & hypogaea & 7.8 & 3.5 & 2.0 & 31.5 & 33.0 & 28.0 & $9.3 * *$ & 12.3 & 63.7 & 33.1 & 13.2 \\
\hline 32 & $\begin{array}{l}\text { ICGV } \\
86031\end{array}$ & vulgaris & 16.6 & 6.5 & 5.5 & 28.5 & $30.0^{*}$ & 31.2 & $7.4 * *$ & $22.3 * *$ & 69.9 & 40.8 & 12.7 \\
\hline & Mean & & 19.10 & 6.52 & 6.22 & 29.84 & 31.63 & 30.90 & 6.77 & 16.68 & 69.22 & 38.63 & 14.74 \\
\hline & C.D. $(5 \%)$ & & 4.92 & 1.19 & 0.86 & 1.19 & 1.43 & 4.10 & 0.95 & 3.34 & 4.42 & 3.69 & 5 \\
\hline & C.D. $(1 \%)$ & & 6.49 & 1.57 & 1.14 & 1.58 & 1.89 & 5.4 & 1.26 & 4.41 & 5.84 & 4.87 & 6.6 \\
\hline & C.V. (\%) & & 12.58 & 8.82 & 7.03 & 1.99 & 2.29 & 6.69 & 7.14 & 9.94 & 3.24 & 4.49 & 17.19 \\
\hline
\end{tabular}

*\&** indicates genotype's significance of superiority for yield and other parameters compared to susceptible check at 5 per cent and 1 per cent level of probability, respectively 


\section{Int.J.Curr.Microbiol.App.Sci (2018) 7(11): 1599-1614}

Table.5 Mean performance of mini core genotypes showing $\leq 4$ score for late leaf spot and rust at 80 DAS

\begin{tabular}{|c|c|c|c|c|c|c|c|c|c|c|c|c|c|}
\hline $\begin{array}{l}\text { Sl. } \\
\text { No. }\end{array}$ & Genotypes & $\begin{array}{l}\text { Botanical } \\
\text { variety }\end{array}$ & $\begin{array}{l}\text { Late } \\
\text { leaf } \\
\text { spot }\end{array}$ & Rust & $\begin{array}{c}\text { Spodoptera } \\
\text { damage }(\%)\end{array}$ & $\begin{array}{c}\text { Days to } \\
\text { initiation } \\
\text { of } \\
\text { flowering }\end{array}$ & $\begin{array}{c}\text { Days to } \\
50 \% \\
\text { flowering }\end{array}$ & $\begin{array}{c}\text { Plant } \\
\text { height } \\
(\mathrm{cm})\end{array}$ & $\begin{array}{c}\text { No. of } \\
\text { primary } \\
\text { branches } \\
\text { per } \\
\text { plant }\end{array}$ & $\begin{array}{l}\text { No. of } \\
\text { pods } \\
\text { per } \\
\text { plant }\end{array}$ & $\begin{array}{c}\text { Shelling } \\
\text { per } \\
\text { cent } \\
(\%)\end{array}$ & $\begin{array}{l}\text { Hundred } \\
\text { seed } \\
\text { weight } \\
\text { (g) }\end{array}$ & $\begin{array}{c}\text { Yield } \\
\text { per } \\
\text { plant } \\
(\mathrm{g})\end{array}$ \\
\hline
\end{tabular}

Late leaf spot resistant genotypes

\begin{tabular}{|c|c|c|c|c|c|c|c|c|c|c|c|c|c|}
\hline 1 & ICG 2381 & hypogaea & 2.5 & 2.5 & 8.8 & 31.5 & $33.0 * *$ & 29.6 & $9.4 * *$ & $23.9 * *$ & 69.0 & $47.6^{* *}$ & 9.3 \\
\hline 2 & ICG 11426 & hypogaea & 2.5 & 4.5 & 12.7 & 31.5 & $33.0 * *$ & 24.3 & 4.6 & $21.2 * *$ & $74.7 * *$ & 42.9 & 14.7 \\
\hline 3 & ICG 12625 & aequatoriana & 2.5 & 6.0 & 16.5 & 30.5 & 31.5 & 30.3 & 4.4 & $14.0 * *$ & 69.3 & $46.2 * *$ & 22.2 \\
\hline 4 & ICG 12370 & hypogaea & 3.5 & 6.5 & 23.5 & 32.5 & $34.0 * *$ & 25.5 & $8.3 * *$ & $14.5^{* *}$ & $71.1 *$ & $45.5^{* *}$ & 18.3 \\
\hline 5 & ICG 875 & hypogaea & 4.0 & 6.5 & 15.3 & 34.0 & $35.0 * *$ & 23.5 & $7.1 * *$ & $22.1 * *$ & $73.5^{* *}$ & $45.4 * *$ & $30.6 * *$ \\
\hline \multicolumn{14}{|c|}{ Rust resistant genotypes } \\
\hline 6 & ICG 2381 & hypogaea & 2.5 & 2.5 & 8.8 & 31.5 & 33.0 & 29.6 & $9.4 * *$ & $23.9 * *$ & 69.0 & $47.6^{* *}$ & 9.3 \\
\hline \multirow[t]{2}{*}{7} & ICG 13723 & hypogaea & 3.5 & 4.5 & 17.7 & 32.5 & 34.0 & 29.3 & $7.6 * *$ & 12.3 & 70.5 & $55.2 * *$ & 16.0 \\
\hline & \multicolumn{13}{|l|}{ Checks } \\
\hline 8 & JL 24 & fastigiata & 7.5 & 6.5 & 43.4 & 29.0 & 30.5 & 34.5 & 5.4 & 9.0 & 66.5 & 40.0 & 18.1 \\
\hline 9 & GPBD 4 & fastigiata & 4.0 & 3.0 & 25.7 & 28.0 & 29.5 & 45.0 & 7.8 & 17.9 & 76.3 & 37.3 & 16.4 \\
\hline & Mean & & 6.52 & 6.22 & 19.10 & 29.84 & 31.63 & 30.90 & 6.77 & 16.68 & 69.22 & 38.63 & 14.74 \\
\hline & C.D. $(5 \%)$ & & 1.19 & 0.86 & 4.92 & 1.19 & 1.43 & 4.10 & 0.95 & 3.34 & 4.42 & 3.69 & 5 \\
\hline & C.D. (1\%) & & 1.57 & 1.14 & 6.49 & 1.58 & 1.89 & 5.4 & 0.48 & 4.41 & 5.84 & 4.87 & 6.6 \\
\hline & C.V. (\%) & & 8.82 & 7.03 & 12.58 & 1.99 & 2.29 & 6.69 & 7.14 & 9.94 & 3.24 & 4.49 & 17.19 \\
\hline
\end{tabular}

$* \& * *$ indicates genotypes are superior to yield and other parameters than susceptible check. 
Iroume and Knauft (1987) also reported negative association between response to biotic stresses and pod yield in case of advanced breeding lines. This suggests the necessity to break negative associations by intensive hybridization or induced mutations followed by selection (Naidu, 2002).

There was significant positive association between late leaf spot and rust in mini core genotypes which could be due to similar host pathogen interactions for late leaf spot and rust pathogens. Earlier, significant positive correlation was reported between late leaf spot and rust in studying diverse groundnut germplasm (Naidu, 2002) and mutants (Motagi, 2001). Late leaf spot incidence had significant negative correlation with days to initiation and fifty per cent flowering in mini core genotypes revealing the late maturing nature of most of the late leaf spot resistant lines. Earlier, Naidu et al., (2016) also reported late maturing nature of multiple stress resistant lines in case of interspecific derivatives of groundnut.

\section{Performance of mini core genotypes}

Among the 184 genotypes in the mini core, only $29(15 \%)$ genotypes showed resistance to Spodoptera litura, 31 (16\%) genotypes showed resistance to late leaf spot and 15 (8 $\%)$ genotypes showed resistance to rust. Among all the botanical varieties, hypogaea had more number of genotypes for resistance to Spodoptera litura (27), late leaf spot (29) and rust (7).

This could be due to the fact that hypogaea had the indeterminate growth habit and also has more growing period. Mini core comprises 10 per cent of the core germplasm and has the desirable diversity for reaction to these major biotic stresses studied due to their diverse genetic and geographic origin (Upadhayaya et al., 2014).
Among the 29 genotypes showing less than 10 per cent leaf damage by Spodoptera litura, eight genotypes viz., ICG 862, ICG 928, ICG 76, ICG 2777, ICG 5016, ICG 12276, ICG 4412 and ICG 9905 recorded significantly higher pod yield per plant compared to high yielding check JL 24 (18.1 g) (Table 4). Among these resistant genotypes, ICG 2777 had high yield per plant (35.1 g) compared to other resistant genotypes. Their high pod yield per plant was mainly due to higher pod number per plant (Table 4). The genotype ICG 2381 was resistant to both late leaf spot and rust diseases in addition to resistance to Spodoptera litura. However, this genotype had low pod yield per plant $(9.3 \mathrm{~g})$. The low yield observed in this multiple biotic stress resistant genotype could be due to diversion of resources towards multiple biotic stress resistance mechanisms. Many reports suggest a strong negative association between resistance and desirable agronomic features (Arulsekar, 1972; Hammons, 1981; Williams et al., 1984; Shew et al., 1995; Gowda et al., 1996). Hence, ICG 2381 can be used as a potential donor for incorporation of multiple stress resistance. Naidu (2002) reported that ICGV 93021, an advanced breeding line had multiple stress resistance with superior number of pods. In mini core collection, Upadhyaya et al., (2014) reported that ICG 12697 (resistant to late leaf spot, rust and $A$. flavus) and ICG 12625 (resistant to late leaf spot, A. flavus and bacterial wilt) had multiple biotic stress resistance along with good yield potential (> $1850 \mathrm{~kg} / \mathrm{ha}$ ).

Among the 184 genotypes of mini core, only five genotypes viz., ICG 2381, ICG 11426, ICG 12625, ICG 12370 and ICG 875 were resistant to late leaf spot (field disease score < 4) (Table 5). Earlier, the genotype ICG 11426 (Upadhyaya et al., 2014; Shivaleela 2012; Sudini et al., 2015) and ICG 12625 (Sujay et $a l ., 2008)$ were reported as resistant to late leaf spot disease among the mini core 
genotypes. Sudini et al., (2015) reported ICG 875 and ICG 12625 as moderately resistant to late leaf spot disease. Among these late leaf spot resistant genotypes, ICG 875 that belong to Virginia runner group had moderate resistance to Spodoptera litura with 15.3 per cent leaf damage and it recorded significantly higher pod yield per plant (30.6 g) compared to the susceptible check JL 24. This genotype could serve as potential donor to incorporate the resistance to late leaf spot into Virginia runner genotypes.

Among 184 genotypes, only 2 genotypes showed resistance to rust (Field disease score $<4$ ). Both genotypes (ICG 2381 and ICG 13723) had significantly higher hundred seed weight i.e., $47.6 \mathrm{~g}$ and $55.2 \mathrm{~g}$, respectively compared to susceptible check JL 24 (40 g) and hence can be potential donor genotype for rust resistance under bold seeded groundnut genotypes.

\section{Multiple biotic stress resistance}

Among the 184 genotypes of mini core genotypes, one germplasm accession, ICG 2381 belonging to botanical variety, hypogea under Virginia runner growth habit had resistance to all the three biotic stresses viz., Spodoptera litura, late leaf spot and rust. Earlier, Upadhayaya et al., (2014) reported that ICG 2381 as resistant to rust and $A$. flavus with good oil quality. Multiple stress resistant genotype, ICG 2381 is a landrace collected from Brazil. It has a purple seeded kernel and also reported to be high oleic type (Ganapati, 2011). Since, this germplasm line had low pod yield and hence can serve as potential donor for multiple biotic stress resistance breeding programme.

In groundnut mini core (184 genotypes), 29 genotypes had less than 10 per cent leaf damage by Spodoptera litura, five genotypes had resistance to late leaf spot and two genotypes had resistance to rust. One germplasm ICG 2381 had resistance to late leaf spot, rust and Spodoptera litura and can be utilised in the resistance backcross breeding programme to develop superior multiple biotic stress resistant genotypes with desirable productivity traits. These biotic resistant genotypes need to be assessed for their resistance reaction under artificial conditions and evaluated over locations and seasons for their consistent performance besides studying their pod features and other quality parameters.

\section{Acknowledgement}

The authors are highly thankful to Dr. H. D. Upadhaya, Genetic Resource Unit, ICRISAT, Hyderabad for supplying groundnut mini core. The authors also thank UAS, Dharwad for financial support in the form of staff research project.

\section{References}

Amin, P. W. 1983. Major field insect pests of groundnut in India and associated crop losses. Indian J. Entomol., 2: 337-344.

Anonymous, 2015.Proc. Annul. Groundnut Workshop. Crop Protection, 13, April, 2015, Junagadh Agric. Univ., Junagadh, India, pp. 22-23.

Apte, U. B., Shetye, V. N., Gawali, M. P. and Jadhav, B. B., 2008. Genetic variability and correlation studies in groundnut (Arachis hypogaea L.). Presented In: Third International Conference for Peanut Genomics and Biotechnology on Advances in Arachis through Genomics and Biotechnology (AAGB- 2008), ICRISAT, Hyderabad (AP), India, 4-8, November, 2008, p. 6.

Arulsekar, S., 1972.Variation pattern in progenies of hybrids between different cultivated forms of groundnut (Arachis hypogaea L.). M. Sc. (Agri.) Thesis, 
Tamil Nadu Agric. Univ., Coimbatore, India.

Astaputre, S. A. and Kulkarni, S., 1996, Estimation of yield losses due to late leaf spot of groundnut. Karnataka $J$. Agric. Sci., 9: 168-172.

Dwivedi, S. L., Pande, S., Rao, J. N. and Nigam, S. N., 2002, Components of resistance to late leaf spot and rust among interspecific derivatives and their significance in a foliar disease resistance breeding in groundnut (Arachis hypogaea L.). Euphytica, 125(1): 81- 88.

FAO, 2016. FAOSTAT data base. http://www.faostat.org.

Ganapati, M., 2011. Genetic variability and mapping of nutritional and oil traits in groundnut (Arachis hypogaea L.). Ph. D. Thesis, Univ. Agric. Sci., Dharwad, Karnataka, India.

Gowda, M. V. C., Prabhu, T. G. and Bhat, R. S., 1996. Variability and association of late leaf spot resistance and productivity in two crosses of groundnut (Arachis hypogaea L.). Crop Improv., 23: 44-48.

Hammons, R. O., 1981. Breeding peanuts for disease resistance: rust and late leaf spot. In: Proc. Peanut Breed. Symp., American Peanut Research Education Society, Richmond, Virginia, 16, July, 1980, pp. 52-58.

Iroume, R. N. and Knauft, D. F., 1987. Heritability and correlations for pod yield and leaf spot resistance in peanut (Arachis hypogaea L.) germplasm. Chinese J. Oil Crop Sci., 20: 31-35.

Khedikar, Y. P., 2008. Molecular tagging and mapping of resistance to late leaf spot and rust in groundnut (Arachis hypogaea L.). Ph. D. Thesis, Univ. Agric. Sci., Dharwad, India.

Khote, A. C., Bendale, V. W., Bhave, S. G. and Patil, P. P., 2009. Genetic variability, heritability and genetic advance in some exotic genotypes of groundnut (Arachis hypogaea L.). Crop Res., 37(2): 186-191.

Kulkarni, K. A., 1989. Bio ecology and management of Spodoptera litura (F.) (Lepidoptera: Noctuidae) on groundnut (Arachis hypogaea L.).Ph. D. Thesis. Univ. Agric. Sci., Dharwad, Karnataka, India.

Madhura, C., 2006. Evaluation and characterization of a mini core subset in groundnut (Arachis hypogaea L.). M. Sc. (Agri.) Thesis, Univ. Agric. Sci., Dharwad.

McDonald, D., Subrahmanyam, P., Gibbons, R. W. and Smith, D. H., 1985.Early and late leaf spots of groundnut. Information Bull. No. 21. International Crops Research Institute for Semi-Arid Tropics, Patancheru, Andhra Pradesh, India.p. 24.

Motagi, B. N., 2001. Genetic analysis of resistance to late leaf spot and rust vis a vis productivity in groundnut (Arachis hypogaea L.). Ph. D. Thesis, Univ. Agric. Sci., Dharwad (India).

Motagi, B. N., Gowda, M. V. C., Patil, A. G. and Shakuntala, N. M., 1997. Induced mutagenesis for breaking the undesirable associations with late leaf spot resistance in groundnut. In: Proc. Third Agric. Sci. Congress, 12-15, March, 1997, Punjab Agric. Univ., Ludhiana, p. 160.

Motagi, B. N., Naidu, G. K., Angadi, C. C. and Gowda, M. V. C., 2014. Potential genotypes for resistance to foliar diseases and productivity in groundnut (Arachis hypogaea L.). Karnataka J. Agric. Sci., 27: 445-448.

Nadaf, H. L. and Habib, A. F., 1987. Studies on genetic variability in bunch groundnut. Mysore J. Agric. Sci., 21 (3): 297-301.

Naidu, G. K., 2002. Genetic analysis of multiple stress resistance and adaptation in groundnut (Arachis hypogaea L.). 
Ph. D. Thesis, Univ. Agri. Sci., Dharwad, Karnataka.

Naidu, G. K., Motagi, B. N. and Gowda, M. V. C., 2016. Potential sources of resistance to biotic stresses in groundnut (Arachis hypogaea L.). Proceedings of National Conference on Hormony with Nature in context of Resource Conservation and Climate Change.2224, October, VinobaBhave Univ., Hazaribag, Jharkand, India.

Nath, U. K. and Alam, M. S., 2002. Genetic variability, heritability and genetic advance of yield and related traits of groundnut (Arachis hypogaea L.). J. Bio Sci., 2(11): 762-764.

Painawadee, S., Joglay, T., Keshmala, C., Akkasaeng, S. and Patanohatti, A., 2009. Heritability and correlation of drought resistance traits and agronomic traits in peanut (Arachis hypogaea L.). Asian J. Pl. Sci., 8(5): 325-334.

Prasad, M. N. R. and Gowda, M. V. C., 2006. Mechanisms of resistance to tobacco cutworm (Spodoptera litura) and their implications to screening for resistance in groundnut. Euphytica, 149: 387-399.

Prasad, M. N. R., 1997. Evaluation of groundnut mutants for resistance to Spodoptera litura and thrips.M. Sc. (Agri.) Thesis, Univ. Agric. Sci., Dharwad, Karnataka, India.

Puranik, S. B., Patil, K. B. G., Patil, N. K., Padaganur, G. M. and Hiremath, R. V., 1973. Fungicidal control of tikka disease of groundnut in Karnataka. Oilseeds J., 3: 24- 28.

Rajashekar, I., 2013. Assessment of genetic diversity and identification of high yielding, drought tolerant and late leaf spot resistant genotypes in a mini-core collection of groundnut (Arachis hypogaea L.). M. Sc. (Agri.) Thesis, Univ. Agric. Sci., Bangalore, Karnataka, India.
Rajgopal, K., Nandagopal, N. and Bhagat, N. R., 1988.Preliminatry screening of Virginia groundnut genotypes against tobacco caterpillar, Spodoptera litura (F.). J. Oilseeds Res., 5: 162-165.

Rao, V. T., Bhadru, D., Murthy, K. G. K. and Bharati, D., 2012. Genetic variability and association among the various characters in groundnut (Arachis hypogaea L.). Int. J. Appl. Bio. Pharmaceutica Techno., 3(3): 337-341.

Raut, R. D., Dhaduk, L. K. and Vachhani, J. H., 2010. Character association and path coefficient analysis in $\mathrm{F}_{2}$ generation of groundnut (Arachis hypogaea L.). Int. J. Agric. Sci., 1: 305-310.

Sandhikar, R. N., Bulbule, S. Y. and Mayee, C. D., 1989. Prediction models for rust epidemic in groundnut. Indian J. Mycol. Pl. Pathol., 19: 60-67.

Savaliya, J. J., Pansuriya, A. G., Sodavadiya, P. R. and Lera, R. L., 2009. Evaluation of inter and interspecific hybrid derivation of groundnut (Arachis hypogaea L.) for yield and its components. Legume Res., 32(2): 129 132.

Sharma, L. K. and Gupta, S. C., 2011. Nature and magnitude of association of pod yield with different morphological characters in parents and hybrids of groundnut (Arachis hypogaea L.), National J. Pl. Improv., 10(2): 129-132.

Shew, B. B., Beute, M. K. and Stalker, H. T., 1995. Towards sustainable peanut production: Progress in breeding for resistance to foliar and soil borne pathogens of peanut. $\mathrm{Pl}$. Disease, 79: 1259-1261.

Shivaleela, K., 2012. Evaluation of a Minicore collection and validation of SSR markers linked to late leaf spot and rust resistance in groundnut (Arachis hypogaea L.). M. Sc. (Agri.) Thesis, Univ. Agric. Sci., Banagalore, Karnataka, India. 
Subrahamanyam, P., McDonald. D., Waliar, F., Reddy, L. J., Nigam, S. N., Gibbons, R. W., Rao, V. R., Singh, A. K., Pande, S., Reddy, P. M. and Rao, P. V. S., 1995. Screening methods and sources of resistance to rust and late leaf spot of groundnut.Information Bull. No. 47, International Crops Research Institute for Semi-Arid Tropics (ICRISAT), Patancheru, Hyderabad (India), pp. 5458.

Subramanyam, P., McDonald. D. and Reddy, L. J., 1990. Resistance to rust and late leaf spot of groundnut at International Crops Research Institute for Semi-Arid Tropics center. In: Proc. $4^{\text {th }}$ Regional Groundnut Workshop South Africa, 1923, March 1990, International Crops Research Institute for Semi-Arid Tropics, Patancheru, Andhra Pradesh, India, pp. 85-92.

Sudini, H., Upadhyaya, H. D., Reddy, S. V., Mangala, U. N., Rathore, A. and Kumar, K. V. K., 2015. Resistance to late leaf spot and rust diseases in ICRISAT's mini core collection of peanut (Arachis hypogaea L.). Australasian Pl. Pathol., 44(5): 557566.

Sujay, V., Kusuma, V. P., Yugandhar, G., Sujatha, B., Gowda, M. V. C. and Upadhyaya, H. D., 2008. Selection of accessions from mini core to improve disease resistance in groundnut. In: $3 r d$ International Conference for Peanut
Genomics and Biotechnology on Advances in Arachis through Genomics and Biotechnology, 4-8, November, 2008, ICRISAT, Patancheru, Andhra Padesh, India, pp. 32-35.

Upadhyaya, H. D., Dwivedi, S. L., Vadez, V., Hamidou, F., Singh, S., Varshney, R. K. and Liao, B., 2014. Multiple resistant and nutritionally dense germplasm identified from mini core collection in peanut. Crop Sci., 54(2): 679-693.

Venkataraman, P., 2001. Variability and correlation studies in groundnut.Crop Res., 21(1): 81-83.

Venkataraman, P., Janakiraman, N. and Madhuprasad, V. L., 2001. Studies on genotype $\times$ environment interaction for oil content and its component characters in groundnut (Arachis hypogaea L.). Crop Res., 22(1): 90-93.

Williams, J. H., Raraj, V. M. and Pal, M., 1984. Physiological studies on foliar disease: varietal differences in response to use of fungicides. In: Groundnut Rust Disease; Proceedings of Discussion Group Meeting, 24-28, September, 1984, International Crop Research Institute for Semi-Arid Tropics, Patancheru, Andhra Pradesh, India, pp. 49-53.

Yugandhar, G., 2005. Evaluation of a mini core set of germplasm in groundnut (Arachis hypogaea L.). M. Sc. (Agri.) Thesis, Univ. Agric. Sci., Dharwad, Karnataka, India.

\section{How to cite this article:}

Mohammad Saleem, A., K. Gopalakrishna Naidu, H.L. Nadaf and Tippannavar, P.S. 2018. Assessment of Groundnut (Arachis hypogaea L.) Mini Core for Resistance to Multiple Biotic Stresses under Hot Spot Location. Int.J.Curr.Microbiol.App.Sci. 7(11): 1599-1614. doi: https://doi.org/10.20546/ijcmas.2018.711.182 